\title{
In vitro Analysis of the Interaction between Human Serum Albumin and Semi-Synthetic Clerodanes
}

\author{
Otávio A. Chaves, ${ }^{a}$ Áurea Echevarria, ${ }^{*, a}$ Andressa Esteves-Souza, ${ }^{a}$ Maria A. M. Maciel ${ }^{b}$ \\ and José C. Netto-Ferreira ${ }^{a, c}$ \\ ${ }^{a}$ Departamento de Química, Universidade Federal Rural do Rio de Janeiro, \\ 23851-970 Seropédica-RJ, Brazil \\ ${ }^{b}$ Departamento de Química, Universidade Federal do Rio Grande do Norte, \\ 59072-970 Natal-RN, Brazil
}

\begin{abstract}
${ }^{c}$ Divisão de Metrologia Química, Instituto Nacional de Metrologia, Qualidade e Tecnologia (INMETRO), 25250-020 Duque de Caxias-RJ, Brazil
\end{abstract}

\begin{abstract}
The interaction between HSA and two semi-synthetic potential anti-cancer agents derived from trans-dehydrocrotonin-methyl-hydrazone (MHDCTN) and phenyl-hydrazone (PHDCTN) was evaluated under physiological conditions at 296, 303 and $310 \mathrm{~K}$ by multi-spectroscopic techniques and molecular docking calculations. Steady state fluorescence quenching indicated a ground state association (static quenching) for both samples; however, the quenching induced by PHDCTN was not essentially static and can be accompanied by a dynamic quenching mechanism. The binding is strong (modified Stern-Volmer binding constant $\left(\mathrm{K}_{\mathrm{a}}\right)$ ca. $\left.10^{5} \mathrm{M}^{-1}\right)$, causing a very weak perturbation on the secondary structure of the protein and there is just one main binding site for both samples (Sudlow's site I). Molecular docking results suggested hydrogen bonding and hydrophobic interactions as the main binding forces for both samples.
\end{abstract}

Keywords: Croton cajucara, trans-dehydrocrotonin derivatives, human serum albumin, spectroscopy, molecular docking

\section{Introduction}

Human serum albumin (HSA) is the most abundant protein in human plasma, being synthesized in the liver and exported as a single non-glycosylated chain, reaching a blood concentration of about $7.0 \times 10^{-4} \mathrm{M} .^{1}$ From the structural point of view, HSA is formed by three homologous domains (named I, II, and III). Each domain is known to be made up by two separate helical subdomains (named A and B) connected by a random coil. In addition, HSA structure presents just one tryptophan residue (Trp-214), contributing significantly for its fluorescence emission, which is used in the albumin-drug binding studies. ${ }^{2}$ The most outstanding property of HSA is its ability to reversibly bind a large variety of endogenous and exogenous ligands and due to this HSA is extensively investigated. When HSA interacts with small molecules such as drugs, this interaction can remarkably affect their absorption, distribution, metabolism and toxicity. At the

*e-mail: echevarr@ufrrj.br same time, the functions and structure of the protein may also change. ${ }^{3}$ Therefore, binding to HSA controls the free active concentration of the drug and may affect considerably the overall pharmacodynamic and pharmacokinetic profile. In other words, studies on albumin-drug binding are important from both theoretical and practical point of view as they allow better understanding of processes underlying drug disposition and elimination, as well as the effect of several pathological states or co-administered drugs on delivery and efficacy. ${ }^{4}$

Croton cajucara Benth (Euphorbiaceae), popularly known as "sacaca", is a widely grown tree of the Amazon region, North of Brazil, used in folk medicine. ${ }^{5}$ The norclerodane trans-dehydrocrotonin ( $t$-DCTN, Figure 1) is the main diterpene present in Croton cajucara. In vitro studies have shown that $t$-DCTN presents potential effects as antigenotoxic, ${ }^{6}$ antileishmanial, ${ }^{7}$ antiulcerogenic, ${ }^{8}$ antitumor, ${ }^{9-11}$ and hypoglycemic. ${ }^{12}$ Recent publication from our group ${ }^{13}$ on the interaction between $t$-DCTN and bovine serum albumin (BSA) demonstrated that there is a spontaneous, weak and entropy-driven association 
between BSA and $t$-DCTN in the ground state, typical of hydrophobic interactions. There is just one main binding site in serum albumin for $t$-DCTN causing a moderate change in the $\alpha$-helix content of the albumin. ${ }^{13}$

Recently, five semi-synthetic clerodanes based on the $t$-DCTN structure were synthesized and assayed against Ehrlich carcinoma and K562 human leukemia cells. Two of them showed remarkable biological activity: methyl-hydrazone (MHDCTN, Figure 1) and phenylhydrazone derivative (PHDCTN, Figure 1), with half maximal inhibitory concentration $\left(\mathrm{IC}_{50}\right) 45.78 \pm 4.35$ and $16.78 \pm 1.42$ for Ehrlich carcinoma, respectively, and $\mathrm{IC}_{50}$ $7.85 \pm 1.49$ and $13.08 \pm 1.12$ for K562 leukemia cells, respectively. ${ }^{14}$ Since the binding ability HSA:drug is one of the steps for a possible pharmacological application and considering that MHDCTN and PHDCTN showed potential anti-cancer activity, the aim of the present study is the continuation of investigation of pharmacological aspects through their interaction with HSA. For this, multispectroscopic techniques (circular dichroism, steady state, time-resolved and synchronous fluorescence) combined with theoretical calculations (molecular docking) were employed.

\section{Experimental}

\section{Materials}

Commercially available HSA, warfarin, ibuprofen, digitoxin and PBS (phosphate-buffered saline) buffer $(\mathrm{pH}=7.4)$ were obtained from Sigma-Aldrich Chemical Company. One tablet of PBS dissolved in $200 \mathrm{~mL}$ of
Millipore water yields concentrations of 0.01, 0.0027 and $0.137 \mathrm{M}$ for phosphate buffer, potassium chloride and sodium chloride, respectively, at pH 7.4 (298 K). Water used in all experiments was Millipore water. Acetonitrile (spectroscopic grade) was obtained from Tedia Ltd. The semi-synthetic clerodanes MHDCTN and PHDCTN were synthesized and characterized according to the literature. ${ }^{14}$

\section{Methods and instruments}

\section{Spectroscopic analysis}

Steady state fluorescence was measured on a Jasco J-815 spectropolarimeter in a quartz cell $(1.0 \mathrm{~cm}$ optical path), employing a thermostated cuvette holder Jasco PFD-425S15F. The circular dichroism (CD) spectra were measured using a spectropolarimeter Jasco J-815, employing the same thermostated cuvette holder as described above. All spectra were recorded with appropriate background corrections.

For the steady state fluorescence measurements, successive aliquots from a stock solution of MHDCTN or PHDCTN $\left(1.00 \times 10^{-3} \mathrm{M}\right.$, in acetonitrile) were added to a $3.0 \mathrm{~mL}$ solution of HSA $\left(1.00 \times 10^{-5} \mathrm{M}\right.$, in PBS), leading to final concentrations of $0.17 ; 0.33 ; 0.50 ; 0.66 ; 0.83 ; 0.99$; 1.15 and $1.32 \times 10^{-5} \mathrm{M}$. The addition was done manually by using a micro syringe. The steady state fluorescence spectra were measured in the $290-450 \mathrm{~nm}$ range, at 296, 303 and $310 \mathrm{~K}$, with $\lambda_{\text {exc }}=280 \mathrm{~nm}$.

CD spectra were recorded in the $200-260 \mathrm{~nm}$ range, at $310 \mathrm{~K}$, for HSA solution $\left(1.00 \times 10^{-6} \mathrm{M}\right.$, in PBS $)$ without and in the presence of MHDCTN or PHDCTN $\left(1.32 \times 10^{-5} \mathrm{M}\right)$.

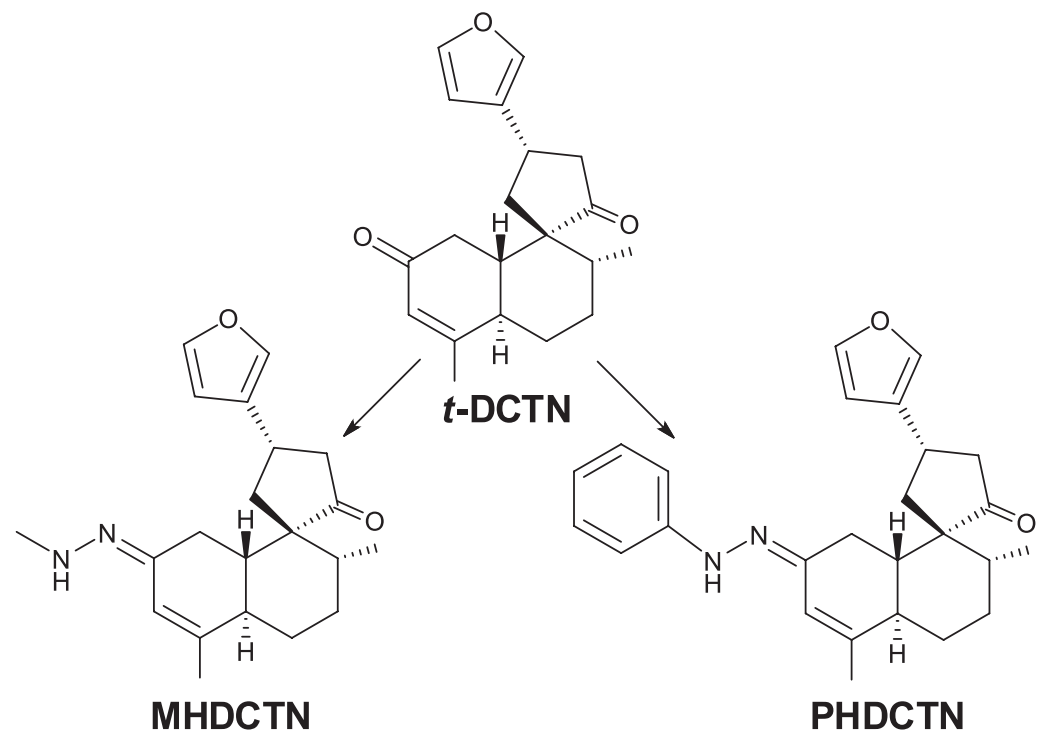

Figure 1. Chemical structure of the natural product $t$-DCTN and its corresponding semi-synthetic derivatives: methyl-hydrazone (MHDCTN) and phenylhydrazone (PHDCTN). 
Time-resolved fluorescence measurements were performed on a model FL920 CD fluorimeter from Edinburgh Instruments equipped with an electrically pumped laser (EPL, $\lambda_{\text {exc }}=280 \pm 10 \mathrm{~nm}$; pulse of $850 \mathrm{ps}$ with energy of $1.8 \mu \mathrm{W}$ per pulse, monitoring emission at $340 \mathrm{~nm})$. The fluorescence decay of a $3.0 \mathrm{~mL}$ solution of HSA $\left(1.00 \times 10^{-5} \mathrm{M}\right.$, in PBS $)$ was measured without and in the presence of $1.32 \times 10^{-5} \mathrm{M}$ of MHDCTN or PHDCTN.

Synchronous fluorescence spectra (SFS) were recorded in a model Xe900 fluorimeter from Edinburgh Instruments. The SFS of a $3.0 \mathrm{~mL}$ HSA solution $\left(1.00 \times 10^{-5} \mathrm{M}\right.$, in PBS $)$ was measured without and in the presence of MHDCTN or PHDCTN, employing the same concentrations used in the steady state fluorescence studies.

Competitive binding studies were carried out using the site markers warfarin, ibuprofen and digitoxin. Each site marker was used in the same concentration of HSA $\left(1.00 \times 10^{-5} \mathrm{M}\right)$ at $310 \mathrm{~K}$. The addition of MHDCTN or PHDCTN to the solution HSA:site marker was done according to the procedure described above for the steady state fluorescence quenching studies.

\section{Molecular docking analysis}

MHDCTN and PHDCTN structures were built and energy-minimized by density functional theory (DFT) calculations (B3LYP potential) with basis set 6-31G*, available in the Spartan'14 program. ${ }^{15}$ The molecular docking studies were performed with the GOLD 5.2 program $^{16}$ (CCDC, Cambridge Crystallographic Data Centre), and the crystallographic structure of HSA used in this simulation was $1 \mathrm{~N} 5 \mathrm{U}$ (PDB code). ${ }^{17}$

Hydrogen atoms were added to the protein according to the data inferred by GOLD 5.2 program ${ }^{16}$ on the ionization and tautomeric states. Docking interaction cavity in the protein was established with a $10 \AA$ radius from the Trp- 214 residue. The number of genetic operations (crossover, migration, mutation) in each docking run used in the search procedure was set to 100,000 . The scoring function used was 'ChemPLP', which is the default function of the GOLD 5.2 program. ${ }^{16}$ More details on the molecular docking procedures can be found in previous publications..$^{18,19}$

\section{Experimental analysis}

\section{Steady state fluorescence analysis}

For the steady state fluorescence quenching data, the inner filter correction was not necessary because at the maximum concentration used of MHDCTN and PHDCTN $\left(1.32 \times 10^{-5} \mathrm{M}\right)$ the $\mathrm{UV}$ absorption is less than 0.1 a.u. at 280 and $340 \mathrm{~nm}$ (Figure S1 in the Supplementary Information (SI) section). However, in order to further clarify this sentence, the inner filter correction (equation S1 in the SI section) was applied for the calculation of three binding constant parameters (Stern-Volmer quenching constant $\left(\mathrm{K}_{\mathrm{SV}}\right)$; bimolecular quenching rate constant $\left(\mathrm{k}_{\mathrm{q}}\right)$; modified Stern-Volmer binding constant $\left(\mathrm{K}_{\mathrm{a}}\right)$ ) as represented in the Figure S2 and Table S1 in the SI section. Since the results calculated without and upon inner filter correction are the same inside the experimental error (Table S1, SI section, and Table 1), in this manuscript the results on the interaction HSA:MHDCTN and HSA:PHDCTN were obtained without inner filter correction.

The steady state fluorescence quenching of HSA in the presence of increasing concentration of MDHCTN or PHDCTN was analyzed by the Stern-Volmer equation (equation 1):20,21

$\frac{\mathrm{F}_{0}}{\mathrm{~F}}=1+\mathrm{k}_{\mathrm{q}} \tau_{0}[\mathrm{Q}]=1+\mathrm{K}_{\mathrm{SV}}[\mathrm{Q}]$

where $\mathrm{F}_{0}$ and $\mathrm{F}$ are the steady state fluorescence intensities of HSA in the absence and presence of a ligand, respectively; [Q], $\mathrm{K}_{\mathrm{SV}}$ and $\mathrm{k}_{\mathrm{q}}$ are the ligand concentration, Stern-Volmer quenching constant and bimolecular quenching rate constant, respectively; $\tau_{0}$ is the lifetime of HSA in the absence of ligand (the measured average value for the fluorescence lifetime of pure albumin was $\left.(5.90 \pm 0.15) \times 10^{-9} \mathrm{~s}\right)$.

In the case where there is more than one possible binding site in a protein, a double logarithmic equation can be applied (equation 2):21

$\log \left(\frac{\mathrm{F}_{0}-\mathrm{F}}{\mathrm{F}}\right)=\log \mathrm{K}_{\mathrm{b}}+\mathrm{n} \log [\mathrm{Q}]$

where $K_{b}$ is the binding constant and $n$ the number of binding sites.

Data from fluorescence quenching experiments were used to calculate the modified Stern-Volmer binding constant $\left(\mathrm{K}_{\mathrm{a}}\right)$ for each ligand, according to equation $3:{ }^{13}$

$\frac{\mathrm{F}_{0}}{\mathrm{~F}_{0}-\mathrm{F}}=\frac{1}{\mathrm{f}[\mathrm{Q}] \mathrm{K}_{\mathrm{a}}}+\frac{1}{\mathrm{f}}$

in which $\mathrm{f}$ is the fraction of the initial fluorescence intensity corresponding to the fluorophore that is accessible to the quencher (f ca. 1.00).

The thermodynamic parameters can be calculated according to the van't Hoff equation (equation 4) and Gibbs free energy equation (equation 5):22

$\ln \mathrm{K}_{\mathrm{a}}=-\frac{\Delta \mathrm{H}^{\circ}}{\mathrm{RT}}+\frac{\Delta \mathrm{S}^{\circ}}{\mathrm{R}}$

$\Delta \mathrm{G}^{\circ}=\Delta \mathrm{H}^{\circ}-\mathrm{T} \Delta \mathrm{S}^{\circ}$ 
in which $\Delta \mathrm{H}^{\circ}, \Delta \mathrm{S}^{\circ}$ and $\Delta \mathrm{G}^{\circ}$ are the enthalpy change, entropy change and Gibbs free energy change, respectively; $\mathrm{T}$ is the temperature $(296,303$ and $310 \mathrm{~K})$ and $\mathrm{R}$ the gas constant $\left(8.3145 \mathrm{~J} \mathrm{~mol}^{-1} \mathrm{~K}^{-1}\right)$.

\section{Circular dichroism analysis}

In $\mathrm{CD}$ analysis, the intensity of the signal can be expressed as mean residue ellipticity (MRE), defined according to equation $6:{ }^{13}$

$\mathrm{MRE}=\frac{\theta}{\left(10 \mathrm{nlC}_{\mathrm{P}}\right)}$

in which $\theta, \mathrm{n}, 1$ and $\mathrm{C}_{\mathrm{P}}$ are the observed $\mathrm{CD}$ (in millidegrees), number of amino acid residues (585 for HSA), ${ }^{23}$ path length of the cell $(1.00 \mathrm{~cm})$ and molar concentration of HSA $\left(1.00 \times 10^{-6} \mathrm{M}\right)$, respectively.

The $\alpha$-helical contents of HSA without and in the presence of a ligand were calculated from the molar residual ellipticity (MRE) values at 208 (equation 7) and $222 \mathrm{~nm}$ (equation 8): ${ }^{18,24}$

$$
\begin{aligned}
& \alpha-\operatorname{helix}(\%)=\frac{\left(-\mathrm{MRE}_{208}-4000\right)}{(33000-4000)} 100 \\
& \alpha-\operatorname{helix}(\%)=\frac{\left(-\mathrm{MRE}_{222}-2340\right)}{30300} 100
\end{aligned}
$$

\section{Results and Discussion}

\section{HSA binding studies}

In pharmacology and pharmacodynamics, proteindrug interactions play a significant role. At molecular level, spectroscopic techniques are valuable methods for the characterization of these interactions due to their high sensitivity, rapidity and easy implementation. ${ }^{3}$ Figure 2 depicts the steady state fluorescence quenching of HSA by the maximum addition of the potential anti-cancer agents under study $\left(1.32 \times 10^{-5} \mathrm{M}\right)$. This quenching process and the absence of significant changes in the maximum for the fluorescence emission $\left(\lambda_{\mathrm{em}}\right.$ ca. $\left.340 \mathrm{~nm}\right)$ indicate that the binding process is probably occurring near the Trp-214 residue present in the HSA structure and that the presence of the quencher does not change the microenvironment around the tryptophan residue. ${ }^{19}$ These results were confirmed by the synchronous fluorescence quenching studies described below.

Steady state fluorescence quenching may involve two different mechanisms, i.e., static and/or dynamic quenching, which exhibit different behavior as a function of
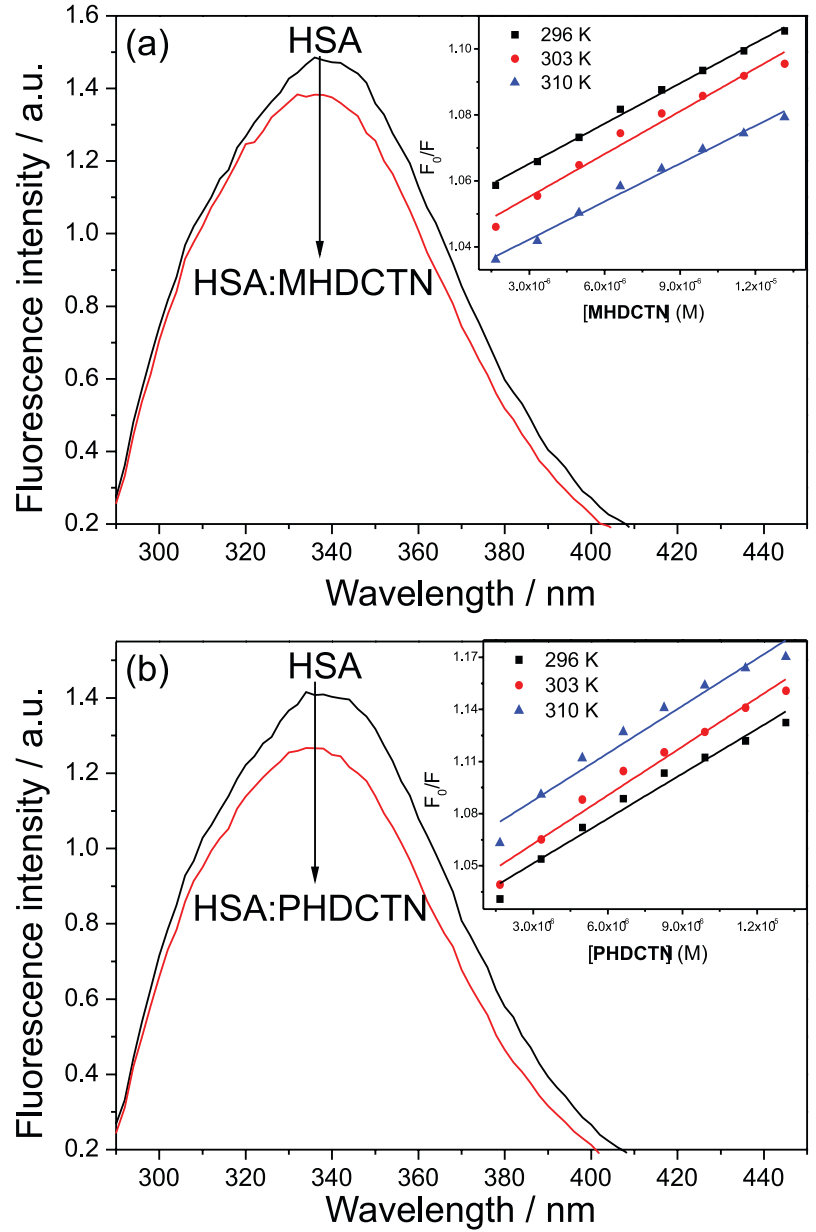

Figure 2. Steady state fluorescence emission of HSA and its quenching by MHDCTN (a) and PHDCTN (b) at $\mathrm{pH}=7.4$ and $310 \mathrm{~K}$. Inset: Stern-Volmer plot of the interaction HSA:MHDCTN (a) and HSA:PHDCTN (b) at 296, 303 and $310 \mathrm{~K}$. [HSA] $=1.00 \times 10^{-5} \mathrm{M}$ and $[\mathrm{MHDCTN}]=[\mathrm{PHDCTN}]=1.32 \times 10^{-5} \mathrm{M}$.

temperature. In general, Stern-Volmer analysis can be used to obtain information about the main fluorescence quenching mechanism (Figure 2, inset). Thus, for both samples (MHDCTN or PHDCTN) the $\mathrm{k}_{\mathrm{q}}$ values listed in Table 1 are higher than the maximum scatter collision quenching constant $\left(2.00 \times 10^{10} \mathrm{M}^{-1} \mathrm{~s}^{-1}\right) .{ }^{25}$ This is a clear indication that the main fluorescence quenching occurs via a static mechanism, i.e., there is a ground state association between HSA and MHDCTN or PHDCTN. ${ }^{26}$ For MHDCTN, $\mathrm{K}_{\mathrm{Sv}}$ values decrease with the increase of temperature, indicating that the main fluorescence quenching mechanism is fully static. However, for PHDCTN the $\mathrm{K}_{\mathrm{Sv}}$ values increased with increasing temperature, which led us to conclude that the fluorescence quenching mechanism for this sample is not essentially static and can be accompanied by a dynamic quenching mechanism. ${ }^{27}$

Time-resolved fluorescence decay can be used to further confirm if the fluorescence quenching mechanism of HSA is occurring via a static and/or a dynamic process. ${ }^{28}$ 
Table 1. Binding constant values $\left(\mathrm{K}_{\mathrm{sv}}, \mathrm{k}_{\mathrm{q}}, \mathrm{n}\right.$ and $\left.\mathrm{K}_{\mathrm{a}}\right)$ for the interaction HSA:MHDCTN and HSA:PHDCTN at 296, 303 and $310 \mathrm{~K}$

\begin{tabular}{|c|c|c|c|c|c|}
\hline Ligand & $\mathrm{T} / \mathrm{K}$ & $\mathrm{K}_{\mathrm{SV}}\left(\times 10^{3}\right) / \mathrm{M}^{-1}$ & $\mathrm{k}_{\mathrm{q}}\left(\times 10^{11}\right) /\left(\mathrm{M}^{-1} \mathrm{~s}^{-1}\right)$ & $\mathrm{n}$ & $\mathrm{K}_{\mathrm{a}}\left(\times 10^{5}\right) / \mathrm{M}^{-1}$ \\
\hline \multirow{3}{*}{ MHDCTN } & 296 & $4.33 \pm 0.26$ & 7.34 & $1.26 \pm 0.11$ & $3.26 \pm 0.26$ \\
\hline & 303 & $4.08 \pm 0.10$ & 6.92 & $1.10 \pm 0.06$ & $2.83 \pm 0.26$ \\
\hline & 310 & $3.84 \pm 0.15$ & 6.50 & $1.03 \pm 0.13$ & $2.32 \pm 0.26$ \\
\hline \multirow{3}{*}{ PHDCTN } & 296 & $8.61 \pm 0.58$ & 14.6 & $0.98 \pm 0.11$ & $1.09 \pm 0.26$ \\
\hline & 303 & $9.09 \pm 0.74$ & 15.4 & $1.01 \pm 0.12$ & $1.45 \pm 0.26$ \\
\hline & 310 & $9.34 \pm 0.66$ & 15.8 & $1.08 \pm 0.12$ & $2.33 \pm 0.26$ \\
\hline
\end{tabular}

HSA: human serum albumin; MHDCTN: methyl-hydrazone trans-dehydrocrotonin; PHDCTN: phenyl-hydrazone trans-dehydrocrotonin; T: temperature; $\mathrm{K}_{\mathrm{sv}}$ : Stern-Volmer quenching constant; $\mathrm{k}_{\mathrm{q}}$ : bimolecular quenching rate constant; $\mathrm{n}$ : number of binding site; $\mathrm{K}_{\mathrm{a}}$ : modified Stern-Volmer binding constant. Note: $\mathrm{r}^{2}$ (coefficient of determination, dimensionless) for $\mathrm{K}_{\mathrm{SV}}$ and $\mathrm{k}_{\mathrm{q}}: 0.9749-0.9955 ; \mathrm{r}^{2}$ for $\mathrm{n}$ : 0.9816-0.9969; $\mathrm{r}^{2}$ for $\mathrm{K}_{\mathrm{a}}: 0.9993-0.9999$.

HSA has two fluorescence lifetimes $\left(\tau_{1}\right.$ and $\left.\tau_{2}\right)$ with $\tau_{2}$ presenting the most relative contribution for the total decay ( $\tau_{1} 23.0 \%$ and $\left.\tau_{2} 77.0 \%\right)$. Fluorescence lifetimes of HSA without $\left(\tau_{1}=1.86 \pm 0.16\right.$ and $\tau_{2}=5.90 \pm 0.15, \chi^{2}=1.105$, Figure 3 , where $\chi^{2}$ (chi squared) measures the goodness of fit of experimental data to a bi-exponential decay for HSA fluorescence) and in the presence of MHDCTN $\left(\tau_{1}=1.82 \pm 0.12\right.$ and $\tau_{2}=5.96 \pm 0.13, \chi^{2}=1.275$, Figure 3$)$ did not show any variation inside the experimental error, indicating that the fluorescence quenching mechanism is static. ${ }^{29}$ On the other hand, in the presence of PHDCTN $\left(\tau_{1}=1.42 \pm 0.11\right.$ and $\tau_{2}=5.42 \pm 0.10, \chi^{2}=1.316$, Figure 3$)$ the time-resolved fluorescence decay changed significantly confirming that, in this case, in addition to the static
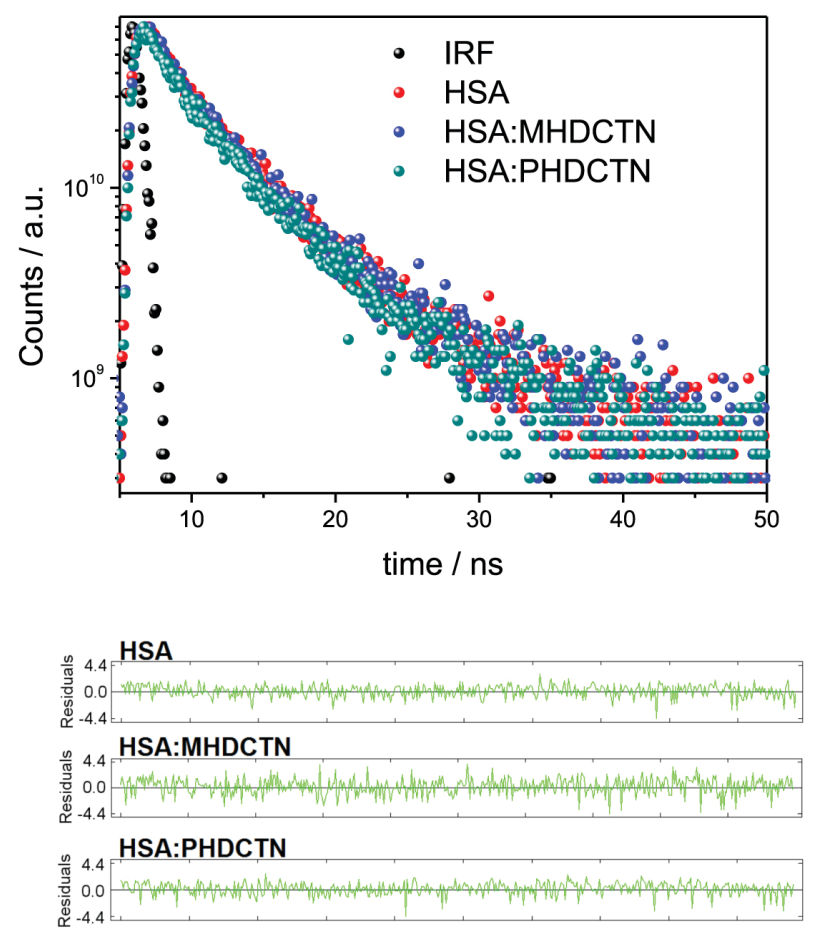

Figure 3. Time-resolved fluorescence decay and its residuals for HSA without and in the presence of MHDCTN or PHDCTN at $\mathrm{pH}=7.4$ and room temperature. $[\mathrm{HSA}]=1.00 \times 10^{-5} \mathrm{M}$ and $[\mathrm{MHDCTN}]=[\mathrm{PHDCTN}]=1.32 \times 10^{-5} \mathrm{M}$. mechanism there is the presence of a dynamic fluorescence quenching mechanism.

HSA has different possible binding sites for different drugs. ${ }^{30}$ In order to evaluate the number of binding sites (n) in HSA, steady state fluorescence quenching data for MHDCTN and PHDCTN were plotted following a double logarithmic equation (equation 2) at 296, 303 and $310 \mathrm{~K}$ (Figure S3, SI section). Since $\mathrm{n}$ values were approximately 1.00 for all temperatures (Table 1), this is a clear indication that a single binding site is available for MHDCTN and PHDCTN on the HSA structure. ${ }^{20}$ Knowing the $n$ value, the binding constant $\left(\mathrm{K}_{\mathrm{a}}\right)$ for the association between HSA and the two semi-synthetic compounds can be calculated employing a modified Stern-Volmer analysis (equation 3$)^{31}$ (Figure S4, SI section). For both quenchers, the $K_{a}$ value is in the order of $10^{5} \mathrm{M}^{-1}$, indicating a strong binding between them and HSA. ${ }^{29-32}$ It is worth to note that results from the literature indicate that $t$-DCTN shows a much weaker binding ability for serum albumin than MHDCTN and PHDCTN..$^{13}$

Since the evaluation of the binding parameters between serum albumin and potential drugs is important to understand its distribution in the human plasma to body tissues and organs, the obtained binding ability between HSA:MHDCTN and HSA:PHDCTN suggests a decrease in the concentration of free molecules in the human plasma. However, the high binding affinity also indicated that the absorption of the potential drugs are feasible, but its distribution to the required tissues will be limited due to the stability of the complex, which in turn adversely affects the pharmacokinetics of the drugs. ${ }^{33,34}$ Comparing these results with those obtained for the natural product $t$-DCTN, the samples MHDCTN and PHDCTN showed better binding ability for HSA, indicating that these dehydrocrotonin derivatives can increase the delivery effectiveness to the site of action.

\section{Thermodynamic analysis}

Several forces such as electrostatic, hydrogen bond, van der Walls, hydrophobic and steric contacts are responsible 
for the interaction between albumin and drugs. The force acting between HSA and semi-synthetic clerodanes was calculated using the van't Hoff plot ${ }^{35}$ (Figure 4) and the values thus obtained are given in Table 2.

From the negative values of $\Delta \mathrm{G}^{\circ}$ (Table 2) one can conclude that the binding is spontaneous for both samples. For MHDCTN, the main contribution for negative $\Delta \mathrm{G}^{\circ}$ arises from $\Delta \mathrm{H}^{\circ}$ and $\Delta \mathrm{S}^{\circ}$, indicating that the binding is enthalpy and entropy driven. Since $\Delta \mathrm{H}^{\circ}<0$ and $\Delta \mathrm{S}^{\circ}>0$,
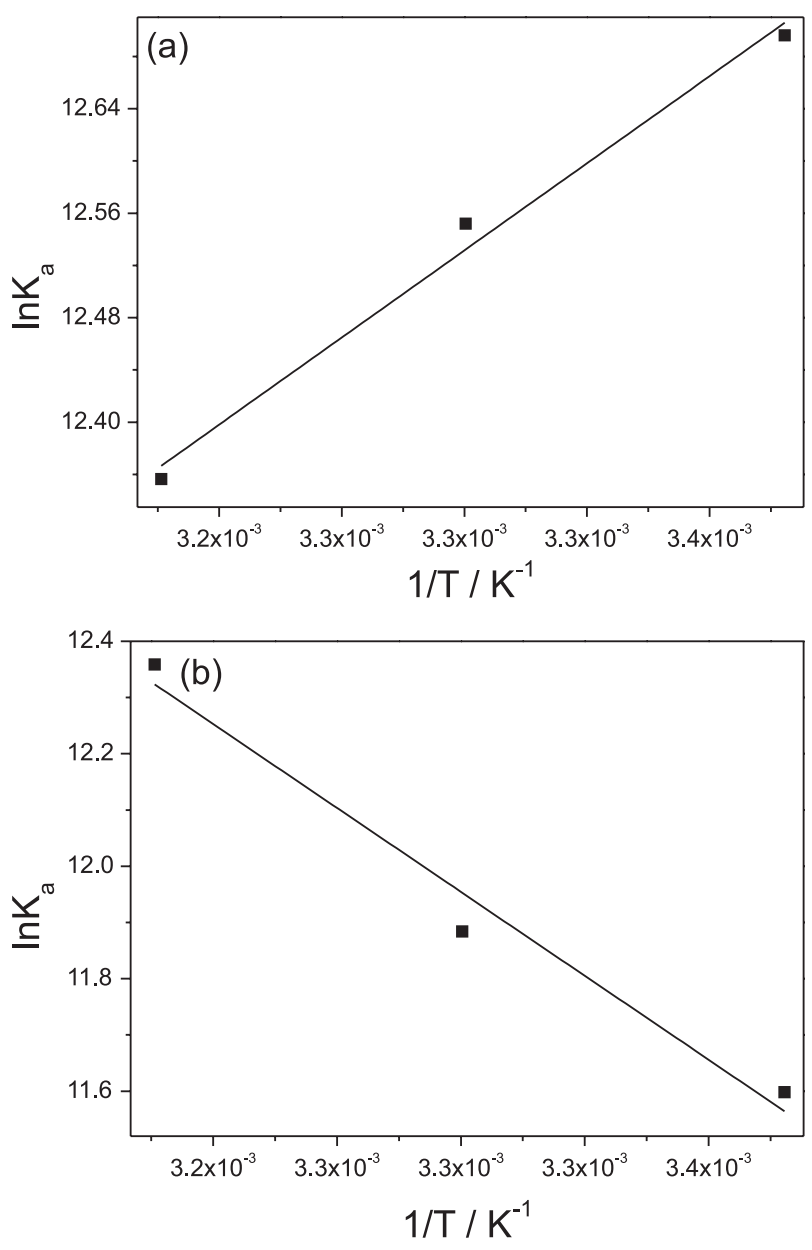

Figure 4. Van't Hoff plot for HSA: MHDCTN (a) and HSA:PHDCTN (b) at 296,303 and $310 \mathrm{~K}$. the involvement of hydrogen bonding and hydrophobic interactions can account for the main binding forces. ${ }^{36} \mathrm{On}$ the other hand, for PHDCTN the main contribution for negative $\Delta \mathrm{G}^{\circ}$ arises from $\Delta \mathrm{S}^{\circ}$, indicating an entropy driven process, ${ }^{37}$ and $\Delta \mathrm{H}^{\circ}>0$ and $\Delta \mathrm{S}^{\circ}>0$ suggest an hydrophobic interaction as the main binding force. ${ }^{38}$ From the literature, the main binding force for the association BSA:t-DCTN is also a hydrophobic interaction, ${ }^{13}$ therefore, the presence of the methyl-hydrazone moiety can change significantly the thermodynamic profile.

\section{Synchronous fluorescence analysis}

Synchronous fluorescence spectra (SFS) analysis has been used to check changes in the microenvironment of the intrinsic fluorescent probes, i.e., the aromatic amino acid residues in $\mathrm{HSA}^{39}$ SFS for pure HSA can offer information about the characteristics of the microenvironment around both the amino acid residue Trp at $\Delta \lambda\left(\lambda_{\text {em }}-\lambda_{\text {ex }}\right)=60 \mathrm{~nm}$ and the Tyr residue at $\Delta \lambda=15 \mathrm{~nm} .{ }^{35}$ Upon successive additions of MHDCTN or PHDCTN to an HSA solution in PBS, the SFS spectral intensity of the latter decreased and did not show any significant change (blue or red shift) at $\Delta \lambda=15$ and $60 \mathrm{~nm}$ (Figure 5). This provides a clear indication that the microenvironment around the aromatic amino acids residues Trp and Tyr does not change with the presence of the potential anti-cancer agents under study. ${ }^{31}$

\section{Perturbation on the secondary structure of the protein}

In order to identify possible structural changes in the HSA structure upon MHDCTN or PHDCTN binding, circular dichroism (CD) experiments were carried out. The CD spectrum of HSA at physiological $\mathrm{pH}$ exhibits two negative bands in the ultraviolet region at $208 \mathrm{~nm}\left(\pi \rightarrow \pi^{*}\right.$ transition) and at $222 \mathrm{~nm}\left(\mathrm{n} \rightarrow \pi^{*}\right.$ transition), which are characteristics of the $\alpha$-helical content. ${ }^{40}$ As can be seen in Figure 6, the decrease in the absorption intensity at 208 and $222 \mathrm{~nm}$ upon MHDCTN or PHDCTN addition

Table 2. Thermodynamic parameters $\left(\Delta \mathrm{H}^{\circ}, \Delta \mathrm{S}^{\circ}\right.$ and $\left.\Delta \mathrm{G}^{\circ}\right)$ for the interaction HSA:MHDCTN and HSA:PHDCTN at 296, 303 and $310 \mathrm{~K}$

\begin{tabular}{|c|c|c|c|c|c|}
\hline Ligand & $\mathrm{T} / \mathrm{K}$ & $\Delta \mathrm{H}^{\circ} /\left(\mathrm{kJ} \mathrm{mol}^{-1}\right)$ & $\Delta \mathrm{S}^{\circ} /\left(\mathrm{kJ} \mathrm{mol}^{-1} \mathrm{~K}^{-1}\right)$ & $\Delta \mathrm{G}^{\circ} /\left(\mathrm{kJ} \mathrm{mol}^{-1}\right)$ & $\mathrm{r}^{2}$ \\
\hline & 296 & & & -31.3 & \\
\hline \multirow[t]{3}{*}{ MHDCTN } & 303 & $-18.5 \pm 1.9$ & $0.0432 \pm 0.006$ & -31.6 & 0.9800 \\
\hline & 310 & & & -31.9 & \\
\hline & 296 & & & -28.5 & \\
\hline \multirow[t]{2}{*}{ PHDCTN } & 303 & $41.4 \pm 6.5$ & $0.236 \pm 0.021$ & -30.1 & 0.9520 \\
\hline & 310 & & & -31.8 & \\
\hline
\end{tabular}

HSA: human serum albumin; MHDCTN: methyl-hydrazone trans-dehydrocrotonin; PHDCTN: phenyl-hydrazone trans-dehydrocrotonin; T: temperature; $\Delta \mathrm{H}^{\circ}$ : enthalpy change; $\Delta \mathrm{S}^{\circ}$ : entropy change; $\Delta \mathrm{G}^{\circ}$ : Gibbs free energy change; $\mathrm{r}^{2}$ : coefficient of determination (dimensionless). 

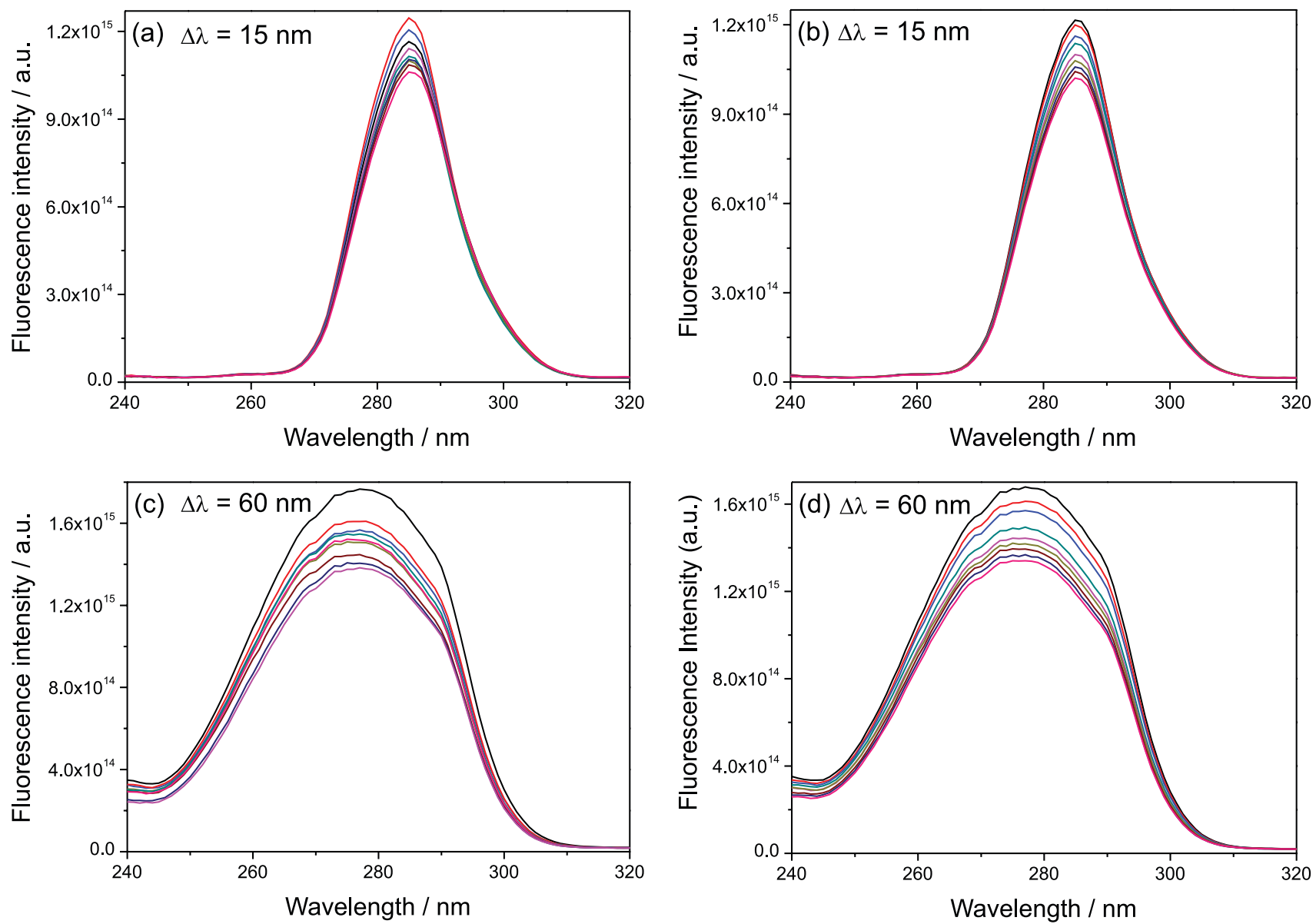

Figure 5. SFS of HSA without and in the presence of $\operatorname{MHDCTN}(\mathrm{a}, \mathrm{c})$ or PHDCTN $(\mathrm{b}, \mathrm{d})$ at $\mathrm{pH}=7.4$ and room temperature. $[\mathrm{HSA}]=1.00 \times 10^{-5} \mathrm{M}$ and $[$ MHDCTN $]=[$ PHDCTN $]=0.17 ; 0.33 ; 0.50 ; 0.66 ; 0.83 ; 0.99 ; 1.15$ and $1.32 \times 10^{-5} \mathrm{M}$.
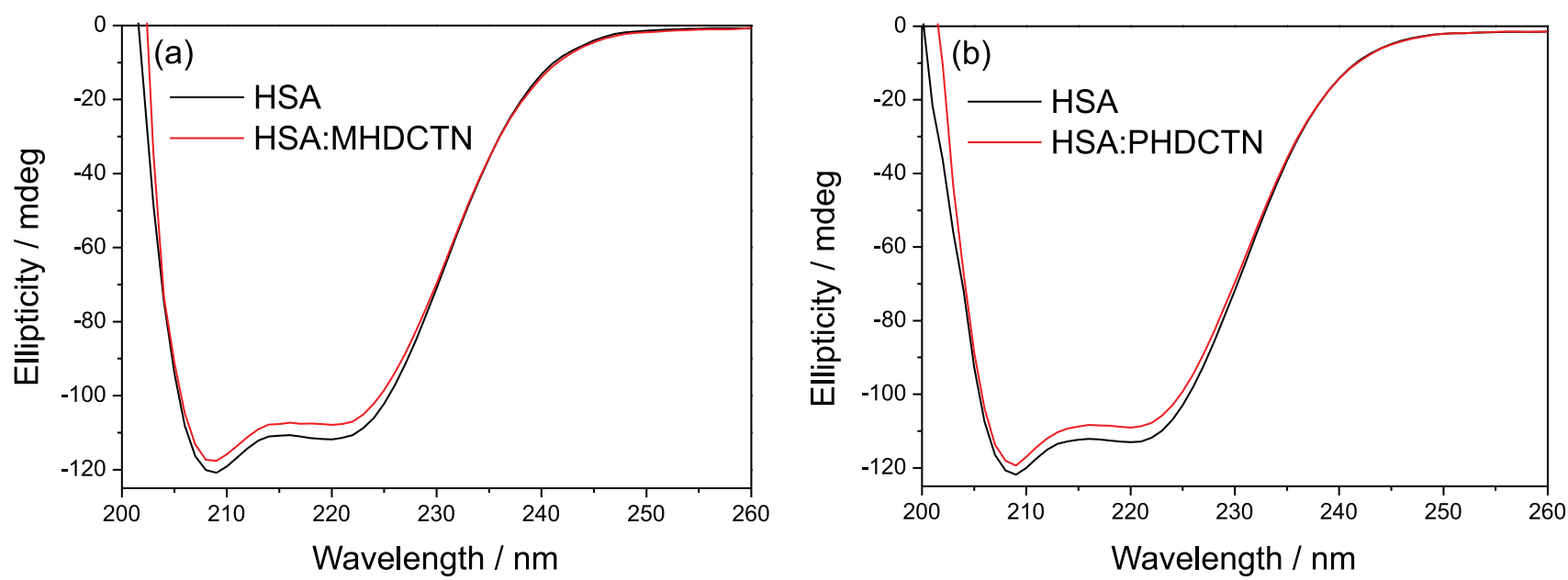

Figure 6. CD spectra for HSA without and in the presence of MHDCTN (a) and PHDCTN (b) at $\mathrm{pH}=7.4$ and $310 \mathrm{~K}$. [HSA] $=1.00 \times 10^{-5} \mathrm{M}$ and $[\mathrm{MHDCTN}]=[\mathrm{PHDCTN}]=1.32 \times 10^{-5} \mathrm{M}$.

indicates the destabilization of the helical structure of HSA, suggesting the occurrence of possible changes in the secondary structure of the protein. ${ }^{31,32}$

Table 3 shows the quantitative percentage of $\alpha$-helical content for HSA in the absence and in the presence of
MHDCTN or PHDCTN at 208 and $222 \mathrm{~nm}$. For pure HSA, the values obtained for the $\alpha$-helical content (in percentage) of its secondary structure were $57.2 \%$ at $208 \mathrm{~nm}$ and $55.1 \%$ at $222 \mathrm{~nm}$. In the presence of MHDCTN a decrease of $1.80 \%$ (at $208 \mathrm{~nm}$ ) and of $2.50 \%$ (at $220 \mathrm{~nm}$ ) on the 
$\alpha$-helical content of the protein was observed. Similar values were obtained for PHDCTN, i.e., $1.80 \%$ at $208 \mathrm{~nm}$ and $2.0 \%$ at $220 \mathrm{~nm}$. These results indicate that both ligands can cause a very weak perturbation on the secondary structure of HSA. ${ }^{41}$

Table 3. The $\alpha$-helical content for HSA without and in the presence of MHDCTN or PHDCTN at $\mathrm{pH}=7.4$ and $310 \mathrm{~K}$

\begin{tabular}{lcc}
\hline \multirow{2}{*}{ Ligand } & \multicolumn{2}{c}{$\alpha$-Helical / } \\
\cline { 2 - 3 } & $208 \mathrm{~nm}$ & $222 \mathrm{~nm}$ \\
\hline HSA & 57.2 & 55.1 \\
HSA:MHDCTN & 55.4 & 52.6 \\
HSA:PHDCTN & 55.8 & 53.1 \\
\hline
\end{tabular}

HSA: human serum albumin; MHDCTN: methyl-hydrazone transdehydrocrotonin; PHDCTN: phenyl-hydrazone trans-dehydrocrotonin.

\section{Competitive binding studies}

HSA has three well-known ligand binding sites, i.e., I, II and III. Site I is located in the subdomain IIA, and is also known as Sudlow's site I (warfarin binding site), while site II is located in the subdomain IIIA, also known as Sudlow's site II (ibuprofen binding site) and site III is located in the subdomain IB (digitoxin binding site). ${ }^{24,42}$ In order to identify the main binding site for the two potential anti-cancer agents in the HSA structure, competitive binding experiments were carried out in the presence of the site markers warfarin, ibuprofen and digitoxin at $310 \mathrm{~K}$. Figure S5 in the SI section depicts the modified Stern-Volmer plots for HSA:MHDCTN and HSA:PHDCTN in the presence of each site marker. As can be seen in Table 4, the $K_{a}$ value changed significantly in the presence of warfarin, i.e., $63.8 \%$ for MHDCTN and $85.0 \%$ for PHDCTN. However, no significant change on the $\mathrm{K}_{\mathrm{a}}$ value was observed for either ibuprofen or digitoxin. This is a clear indication that the main ligand binding site for both samples is the Sudlow's site I in subdomain IIA, where the Trp-214 residue can be found. ${ }^{18,31}$

\section{Molecular docking studies}

Commonly, molecular docking analysis is applied for prediction of protein-drug interactions, as well as on the orientation and conformation of a drug within a biomacromolecule receptor. This useful method allows the identification of protein-inhibitor interactions as well as the structural features of the active site of the protein. ${ }^{43,44}$ From the steady state fluorescence quenching studies, it is known that MHDCTN and PHDCTN can bind near the Trp-214 amino acid residue, located in subdomain IIA (Sudlow's site I). ${ }^{19,27}$ In order to offer a molecular level explanation on the binding ability to HSA of these two potential anticancer agents, molecular docking studies were carried out.

Molecular docking results suggest hydrogen bonding and hydrophobic interactions as the main binding forces for the association HSA:MHDCTN and HSA:PHDCTN. Although both ligands show considerably high kinetic volume, they can be accommodated inside the protein cavity of HSA and close to its intrinsic fluorophore, i.e., the tryptophan residue (Figure 7a).

Figure $7 \mathrm{~b}$ depicts the best docking pose for HSA:MHDCTN. The oxygen atom of the carbonyl group in the ligand structure is an acceptor for hydrogen bonding with Lys-194 residue, within a distance of $2.10 \AA$, while the furan group of the ligand is hydrogen bonded to Lys198, within a distance of $3.10 \AA$. The amino acids residues Leu-197, Trp-214 and Val-342 can interact via hydrophobic forces with the ligand structure.

The best docking pose for HSA:PHDCTN shows that the oxygen atom of the carbonyl group in the ligand structure is an acceptor for hydrogen bonding with the guanidinium group of Arg-221, within a distance of $3.10 \AA$, while the furan group of the ligand is hydrogen bonding with the intrinsic HSA fluorophore Trp-214 residue, within a distance of $3.70 \AA$ (Figure 7c). The amino acids residues Leu-197, Val-342 and Leu-480 can interact via hydrophobic forces with the ligand structure.

Table 4. Modified Stern-Volmer binding constant value $\left(\mathrm{K}_{\mathrm{a}}\right)$ for the interaction HSA:MHDCTN and HSA:PHDCTN in the presence of several site markers, at $310 \mathrm{~K}$

\begin{tabular}{lcccc}
\hline Ligand & Site marker & $\mathrm{K}_{\mathrm{a}}\left(\times 10^{5}\right) / \mathrm{M}^{-1}$ & Change / & $\mathrm{r}^{2}$ \\
\hline \multirow{2}{*}{ MHDCTN } & warfarin & $0.84 \pm 0.02$ & 63.8 & 0.9999 \\
& ibuprofen & $2.23 \pm 0.26$ & 3.88 & 0.9996 \\
\hline \multirow{2}{*}{ PHDCTN } & digitoxin & $1.93 \pm 0.26$ & 16.8 & 0.9996 \\
& warfarin & $0.35 \pm 0.02$ & 85.0 & 0.9993 \\
& ibuprofen & $2.00 \pm 0.26$ & 14.2 & 0.9995 \\
\hline
\end{tabular}

HSA: human serum albumin; MHDCTN: methyl-hydrazone trans-dehydrocrotonin; PHDCTN: phenyl-hydrazone trans-dehydrocrotonin; $\mathrm{K}_{\mathrm{a}}$ : modified Stern-Volmer binding constant; $\mathrm{r}^{2}$ : coefficient of determination (dimensionless). 


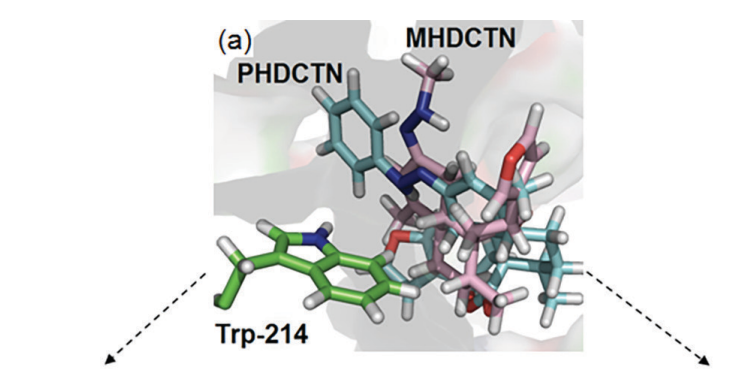

(b) Leu-197

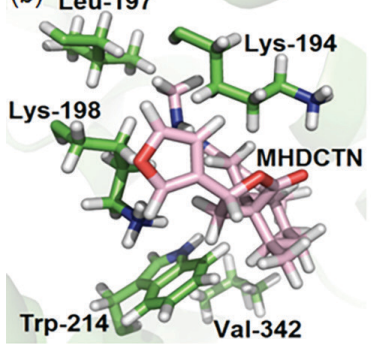

(c)

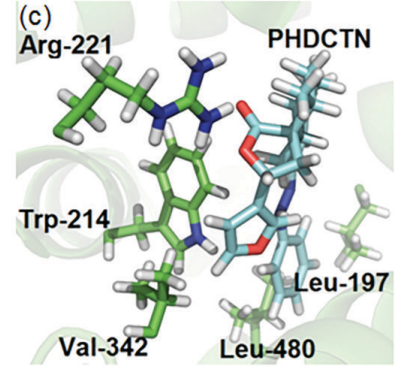

Figure 7. (a) Representation of the molecular surface of HSA in Sudlow's site I (ChemPLP function). Best score pose for HSA:MHDCTN (b) and HSA:PHDCTN (c). Select amino acids residues, MHDCTN and PHDCTN are represented in green, light pink and cyan, respectively. Hydrogen: white; oxygen: red; nitrogen: dark blue.

\section{Conclusions}

From steady state fluorescence quenching data, $\mathrm{k}_{\mathrm{q}}$ ca. $10^{11}-10^{12} \mathrm{M}^{-1} \mathrm{~s}^{-1}$ indicates that a static mechanism is the main fluorescence quenching mechanism, i.e., there is a ground state association between HSA and the two potential anti-cancer agents MHDCTN and PHDCTN. $\mathrm{K}_{\mathrm{SV}}$ values and time-resolved fluorescence decay confirmed the static mechanism for MHDCTN and show that the fluorescence quenching mechanism for PHDCTN is not essentially static and can be accompanied by a dynamic quenching. Fluorescence studies also revealed that there is just one main binding site in the protein structure for interaction with the ligand, which was identified as Sudlow's site I (subdomain IIA) by competitive binding experiments. $\mathrm{K}_{\mathrm{a}}$ ca. $10^{5} \mathrm{M}^{-1}$ indicates a strong binding between HSA and MHDCTN or PHDCTN, with the thermodynamic parameters being in total agreement with the spontaneity of the binding. This binding does not perturb the microenvironment around Trp and Tyr residues, however, it can cause a weak perturbation on the secondary structure of the protein. Molecular docking results suggest hydrogen bonding and hydrophobic interactions as the main binding forces. MHDCTN can interact with the amino acid residues Leu-194, Leu-197, Leu-198, Trp-214 and Val-342, while PHDCTN can interact with Leu-197, Trp-214, Arg-221, Val-342 and Leu-480 residues. Comparing these results with those obtained to the natural product $t$-DCTN, the samples MHDCTN and PHDCTN showed better binding ability for HSA, indicating that the proposed derivative of the dehydrocrotonin moiety can help the delivery effectiveness to the site of action.

\section{Supplementary Information}

Supplementary data (UV-Vis spectra, inner filter correction, Stern-Volmer, double logarithmic and modified Stern-Volmer plots) are available free of charge at http:// jbcs.sbq.org.br as PDF file.

\section{Acknowledgments}

This research was supported by the Brazilian funding agencies: Coordenação de Aperfeiçoamento de Pessoal de Nível Superior (CAPES), Conselho Nacional de Desenvolvimento Científico e Tecnológico (CNPq) and Fundação de Amparo à Pesquisa do Estado do Rio de Janeiro (FAPERJ). The authors also acknowledge Prof Dr Carlos M. R. Sant' Anna (Chemistry Department, UFRRJ) for molecular docking facilities and Prof Dr Nanci Camara de Lucas Garden (Chemistry Institute, UFRJ) for the timeresolved and synchronous fluorescence facilities.

\section{References}

1. Fasano, M.; Curry, S.; Terreno, E.; Galliano, M.; Fanali, G.; Narciso, P.; Notari, S.; Ascenzi, P.; IUBMB Life 2005, 57, 787.

2. Sakurai, Y.; Ma, S. F.; Watanabe, H.; Yamaotsu, N.; Hirono, S.; Kurono, Y.; Kragh-Hansen, U.; Otagiri, M.; Pharm. Res. 2004, $21,285$.

3. Shahraki, S.; Shiri, F.; Saeidifar, M.; Inorg. Chim. Acta 2017, $463,80$.

4. Zhivkova, Z. D.; Curr. Pharm. Des. 2015, 21, 1817.

5. di Stasi, L. C.; Hiruma, C. A.; Guimarães, E. M.; Santos, C. M.; Fitoterapia 1994, 65, 529.

6. Souza-Brito, A. R. M.; Rodriguez, J. A.; Hiruma-Lima, C. A.; Haun, M.; Nunes, D. S.; Planta Med. 1998, 64, 126.

7. Rosa, M. S. S.; Mendonça-Filho, R. R.; Bizzo, H. R.; Rodrigues, I. A.; Soares, R. M. A.; Souto-Padron, T.; Alviano, C. S.; Lopes, A. H. C. S.; Antimicrob. Agents Chemother. 2003, 47, 1895.

8. Rodriguez, J. A.; Hiruma-Lima, C. A.; Brito, A. R. M. S.; Hum. Exp. Toxicol. 2004, 23, 455.

9. Qiu, M.; Cao, D.; Gao, Y.; Li, S.; Zhu, J.; Yang, B.; Zhou, L.; Zhou, Y.; Jin, J.; Zhao, Z.; Fitoterapia 2016, 108, 81.

10. Jullian, V.; Bonduelle, C.; Valentin, A.; Acebey, L.; Duigou, A.-G.; Prévost, M.-F.; Sauvain, M.; Bioorg. Med. Chem. Lett. 2005, 15, 5065.

11. Freire, A. C. G.; Assis, C. F.; Frick, A. O.; Melo, P. S.; Haun, M.; Aoyama, H.; Durán, N.; Sauer, M. M.; Kállas, E. G.; Ferreira, C. V.; Leuk. Res. 2003, 27, 823. 
12. Farias, R. A. F.; Planta Med. 1997, 66, 558

13. Chaves, O. A.; Soares, B. A.; Maciel, M. A. M.; Sant'Anna, C. M. R.; Netto-Ferreira, J. C.; Cesarin-Sobrinho, D.; Ferreira, A. B. B.; J. Braz. Chem. Soc. 2016, 27, 1858.

14. Esteves-Souza, A.; Pissinate, K.; Maciel, M. A. M.; Echevarria, A.; J. Braz. Chem. Soc. 2018, 29, 133.

15. Hehre, W. J.; A Guide to Molecular Mechanics and Quantum Chemical Calculations; Wavefunction, Inc.: Irvine, USA, 2003.

16. http://www.ccdc.cam.ac.uk/solutions/csd-discovery/ components/gold/, accessed in December 2017.

17. Wardell, M.; Wang, Z.; Ho, J. X.; Robert, J.; Ruker, F.; Ruble, J.; Carter, D. C.; Biochem. Biophys. Res. Commun. 2002, 291, 913.

18. Chaves, O. A.; de Oliveira, C. H. C. S.; Ferreira, R. C.; Pereira, R. P.; de Melos, J. L. R.; Rodrigues-Santos, C. E.; Echevarria, A.; Cesarin-Sobrinho, D.; J. Fluorine Chem. 2017, 199, 103.

19. Chaves, O. A.; Amorim, A. P. O.; Castro, L. H. E.; Sant'Anna, C. N. R.; de Oliveira, M. C. C.; Cesarin-Sobrinho, D.; NettoFerreira, J. C.; Ferreira, A. B. B.; Molecules 2015, 20, 19526.

20. Cao, H.; Yi, Y.; Biometals 2017, 30, 529.

21. Mukhopadhyay, S.; Mitra, K.; Paitandi, R. P.; Singh, R. S.; Singh, S.; Ray, B.; Pandey, D. S.; ChemistrySelect 2017, 2, 6000 .

22. Al-Shabib, N. A.; Khan, J. M.; Ali, M. S.; Al-Lohedan, H. A.; Khan, M. S.; Al-Senaidy, A. M.; Husain, F. M.; Shamsi, M. B.; J. Mol. Liq. 2017, 230, 557.

23. Matei, I.; Hillebrand, M.; J. Pharm. Biomed. Anal. 2010, 51, 768.

24. Yuan, L.; Liu, M.; Sun, B.; Liu, J.; Wei, X.; Wang, Z.; Wang, B.; Han, J.; J. Mol. Liq. 2017, 248, 330.

25. Rub, M. A.; Khan, J. M.; Azum, N.; Asiri, A. M.; J. Mol. Liq. 2017, 241, 91.

26. Tian, Z. Y.; Song, L. N.; Zhao, Y.; Zang, F. L.; Zhao, Z. H.; Chen, N. H.; Xu, X. J.; Wang, C. J.; Molecules 2015, 20, 16491.

27. Chaves, O. A.; Mathew, B.; Cesarin-Sobrinho, D.; Lakshminarayanan, B.; Joy, M.; Mathew, G. E.; Suresh, J.; Netto-Ferreira, J. C.; J. Mol. Liq. 2017, 242, 1018.
28. Helms, M. K.; Peterson, C. E.; Bhagavan, N. V.; Jameson, D. M.; FEBS Lett. 1997, 408, 67.

29. Farooqi, M. J.; Penick, M. A.; Negrete, G. R.; Brancaleon, L.; Protein J. 2013, 32, 1.

30. Alanazi, A. M.; Abdelhameed, A. S.; Bakheit, A. H.; Darwish, I. A.; J. Mol. Liq. 2017, 238, 3.

31. Chaves, O. A.; Cesarin-Sobrinho, D.; Sant'Anna, C. M. R.; de Carvalho, M. G.; Suzart, L. R.; Catunda-Junior, F. E. A.; NettoFerreira, J. C.; Ferreira, A. B. B.; J. Photochem. Photobiol., A 2017, 336, 32.

32. Kumari, M.; Maurya, J. K.; Singh, U. K.; Khan, A. B.; Ali, M.; Singh, P.; Spectrochim. Acta, Part A 2014, 124, 349.

33. Naveenraj, S.; Anandan, S.; J. Photochem. Photobiol., C 2013, $14,53$.

34. Ercelen, S.; Klymchenko, A. S.; Mély, Y.; Demchenko, A. P.; Int. J. Biol. Macromol. 2005, 35, 231.

35. Ali, M. S.; Al-Lohedan, H. A.; J. Mol. Liq. 2014, 197, 124.

36. Ali, M. S.; Al-Lohedan, H. A.; J. Mol. Liq. 2017, 236, 232.

37. Chaves, O. A.; Jesus, C. S. H.; Cruz, P. F.; Sant'Anna, C. M. R.; Brito, R. M. M.; Serpa, C.; Spectrochim. Acta, Part A 2016, 169, 175.

38. Bi, S.; Yan, L.; Pang, B.; Wang, Y.; J. Lumin. 2012, 132, 132.

39. Karthiga, D.; Chandrasekaran, N.; Mukherjee, A.; J. Mol. Liq. 2017, 232, 251.

40. Chaves, O. A.; Teixeira, F. S. M.; Guimarães, H. A.; Braz-Filho, R.; Vieira, I. J. C.; Sant'Anna, C. M. R.; Netto-Ferreira, J. C.; Cesarin-Sobrinho, D.; Ferreira, A. B. B.; J. Braz. Chem. Soc. 2017, 28, 1229.

41. Sun, Z.; Xu, H.; Cao, Y.; Wang, F.; Mi, W.; J. Mol. Liq. 2016, $219,415$.

42. Sudlow, G.; Birkett, D. J.; Wade, D. N.; Mol. Pharmacol. 1976, 12, 1052.

43. Meng, X.-Y.; Zhang, H.-X.; Mezei, M.; Cui, M.; Curr. Comput.Aided Drug Des. 2011, 7, 146.

44. Shahraki, S.; Shiri, F.; Mansouri-Torshizi, H.; Shahraki, J.; J. Iran. Chem. Soc. 2016, 13, 723.

Submitted: December 18, 2017

Published online: March 19, 2018 